University of Nebraska - Lincoln

DigitalCommons@University of Nebraska - Lincoln

2-1-1996

\title{
Low energy electron scattering from $\mathrm{CH}_{3} \mathrm{Cl}$
}

X. Shi

University of Nebraska - Lincoln

V.K. Chan

University of Nebraska - Lincoln

Gordon A. Gallup

University of Nebraska-Lincoln, ggallup1@unl.edu

Paul Burrow

University of Nebraska - Lincoln, pburrow1@unl.edu

Follow this and additional works at: https://digitalcommons.unl.edu/physicsburrow

Part of the Physics Commons

Shi, X.; Chan, V.K.; Gallup, Gordon A.; and Burrow, Paul, "Low energy electron scattering from $\mathrm{CH}_{3} \mathrm{Cl}$ (1996). Paul Burrow Publications. 10.

https://digitalcommons.unl.edu/physicsburrow/10

This Article is brought to you for free and open access by the Research Papers in Physics and Astronomy at DigitalCommons@University of Nebraska - Lincoln. It has been accepted for inclusion in Paul Burrow Publications by an authorized administrator of DigitalCommons@University of Nebraska - Lincoln. 


\title{
Low energy electron scattering from $\mathrm{CH}_{3} \mathrm{Cl}$
}

\author{
X. Shi, V. K. Chan, G. A. Gallup, and P. D. Burrow \\ Department of Physics and Astronomy, University of Nebraska, Lincoln, Nebraska 68588
}

(Received 12 July 1995; accepted 25 October 1995)

\begin{abstract}
Differential cross section measurements for the elastic scattering of electrons from $\mathrm{CH}_{3} \mathrm{Cl}$ at energies from 0.5 to $9.5 \mathrm{eV}$ are reported for scattering angles of $30^{\circ}$ and $100^{\circ}$. The angular scattering dependence is determined at selected energies over this range. At energies below $1.0 \mathrm{eV}$, the cross sections are in excellent agreement with calculations using the Born dipole approximation. At large angles and higher energies, the scattering is dominated by a ${ }^{2} A_{1}$ temporary negative ion state near $3.5 \mathrm{eV}$. Energy loss data at 3.5, 5.0, and $8.5 \mathrm{eV}$ are reported and the relative contributions of various vibrational modes determined. Differential cross sections for vibrational excitation of the $v_{3}\left(a_{1}\right)^{\mathrm{C}-\mathrm{Cl}}$ and $v_{4}(e)^{\mathrm{CH}}$ stretching modes have been measured. The latter reveals a broad shape resonance of ${ }^{2} E$ symmetry peaking near $5.5 \mathrm{eV}$. Angular distributions for excitation of these same modes are also reported. Using fittings to the vibrational excitation functions, the resonance parameters have been extracted and used in a mixed semiempirical $a b$ initio calculation to compute the relative strengths of the vibrational energy loss peaks. These compare favorably with the results of the experiment. Elastic cross sections integrated over angle are reported for low energies. They are substantially larger than the results from recent measurements of the total cross section. (C) 1996 American Institute of Physics. [S0021-9606(96)01405-1]
\end{abstract}

\section{INTRODUCTION}

Methyl chloride is the simplest chloro-hydrocarbon and as such plays a fundamental role in our understanding of more complex chlorinated hydrocarbons. In particular, the characteristics of the temporary negative ion states of this compound are of importance since they affect the vibrational excitation and dissociative attachment cross sections under electron impact. In an earlier communication, ${ }^{1}$ we reported energy loss spectra in $\mathrm{CH}_{3} \mathrm{Cl}$ and $\mathrm{CD}_{3} \mathrm{Cl}$ and relative excitation functions for several vibrational modes of $\mathrm{CH}_{3} \mathrm{Cl}$ that resolved an existing controversy about the locations and symmetries of the temporary negative ion states. In the present work, we present absolute differential cross sections for elastic scattering and excitation of selected vibrational modes over an energy range from approximately 1 to $10 \mathrm{eV}$. In addition, using these data, we have reevaluated the energies and lifetimes of the two lower temporary negative ion states. With these more accurate empirical parameters, the lowest order mixed semiempirical ab initio theory for vibrational excitation of polyatomic molecules given by Gallup ${ }^{2}$ is used, for comparison with experiment, to determine the relative strengths for excitation of the vibrational modes of $\mathrm{CH}_{3} \mathrm{Cl}$ at an energy of $3.5 \mathrm{eV}$, where the two resonances overlap.

\section{EXPERIMENT}

The electron scattering apparatus employs hemispherical deflectors and is similar in design to those of Comer and Read $^{3}$ and Boness and Schulz. ${ }^{4}$ The electron optics are scaled to use 1 in diameter electrodes spaced by $1 / 16$ in diameter sapphire balls. All lenses and hemispheres are made of molybdenum, with support components constructed of ti- tanium and aluminum to avoid residual magnetic fields. A cylindrical magnetic shield reduces the earth's magnetic field below $2 \mathrm{mG}$ at the electron beam.

The most novel aspect of the apparatus is the gas handling system ${ }^{5,6}$ used in conjunction with the relative flow measurements. ${ }^{7}$ The details are presented elsewhere. ${ }^{5}$ Briefly, the target gas and the cross section calibrant, usually $\mathrm{He}$, are both brought into the vacuum chamber continuously. An internal four-port switch (Hamilton Co.) directs the target gas to a needle whose output crosses the electron beam, and the calibrant into the background, or the reverse. The switch is controlled from the outside through a rotatable feedthrough. At the cost of somewhat higher background pressure, this method avoids the shifts in contact potential which are encountered when one gas is removed and another introduced into the vacuum chamber and contributes greatly to the overall stability of the apparatus.

In previous work ${ }^{5}$ we demonstrated that our measured cross sections in $\mathrm{Ne}$ at low energy were in excellent accord with theory ${ }^{8}$ and with the results of a swarm data analysis, ${ }^{9}$ and thus we argued that neon was well suited to serve as a secondary standard for verifying the operation of an electron scattering apparatus at low energy. Gulley et al. ${ }^{10}$ have further examined this proposal in detail. In our case, tests in $\mathrm{Ne}$ were carried out before and near the conclusion of the measurements on $\mathrm{CH}_{3} \mathrm{Cl}$ at selected energies and angles, and agreement with the theoretical results of $\mathrm{Saha}^{8}$ was found to within $3 \%$. The statistical errors in these check were approximately $5-6 \%$. These results provide support for believing that systematic errors in the present measurements are relatively small.

For the details of our use of the relative flow method, the reader is referred to Shi and Burrow. ${ }^{5}$ In the present work, the gas kinetic diameter of $\mathrm{CH}_{3} \mathrm{Cl}$ was taken to be $4.182 \times 10^{-8} \mathrm{~cm} .{ }^{11}$ A flow rate ratio of approximately 0.077 


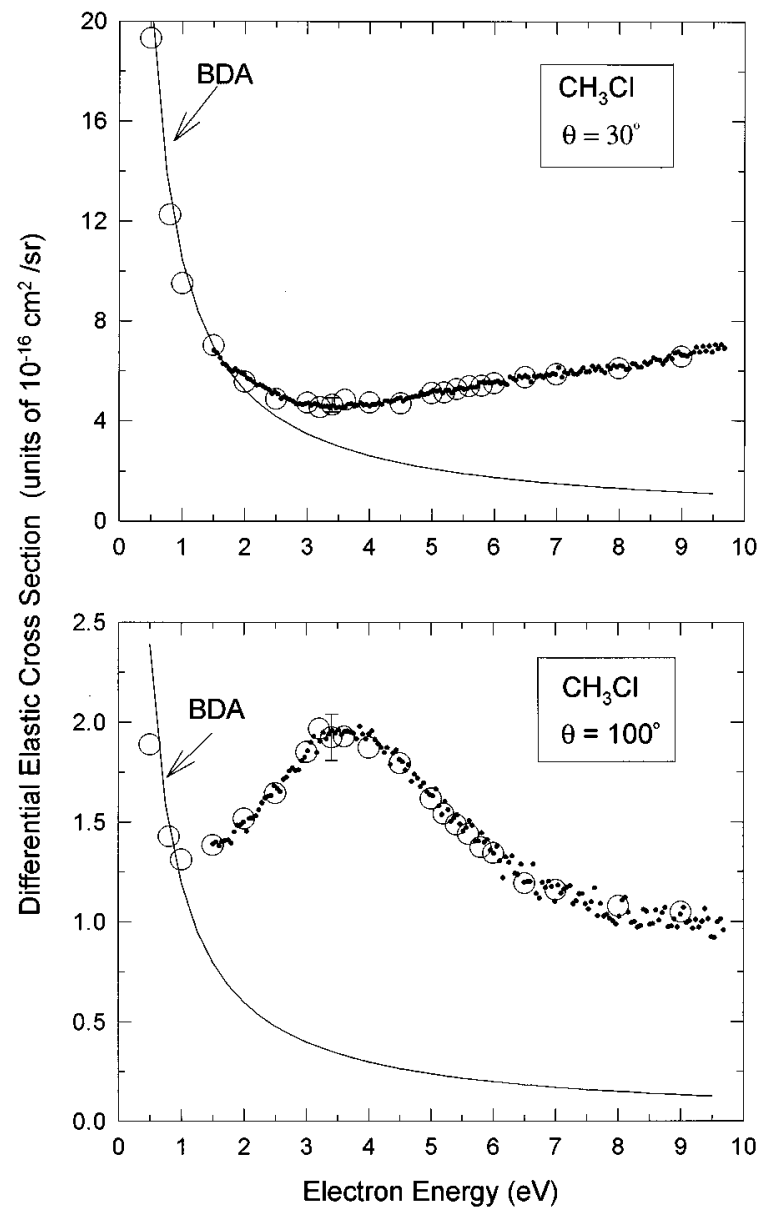

FIG. 1. The differential cross section for elastic scattering of electrons from $\mathrm{CH}_{3} \mathrm{Cl}$ as a function of energy at $30^{\circ}$ (upper panel) and $100^{\circ}$ (lower panel). The solid line marked BDA shows the contribution calculated by use of the Born dipole approximation. The data shown as open circles are individually normalized to the He cross section. Data shown as solid dots were taken in a relative mode and normalized at only a single energy (see text).

for $\mathrm{CH}_{3} \mathrm{Cl}$ to $\mathrm{He}$ was used to ensure that the mean free paths of both gases at the needle were the same. ${ }^{7}$ Energy scale calibrations were carried out using a mixture of $\mathrm{N}_{2}$ and $\mathrm{CH}_{3} \mathrm{Cl}$ and referring to the resonance peaks in the $2 \mathrm{eV}$ region given by Rohr. ${ }^{12}$ The electron energy resolution was typically $28-30 \mathrm{meV}$ full width at half maximum (FWHM) as observed in the elastically scattered beam.

The absolute cross section data presented here as large open circles were obtained by normalization at each energy and angle to the He elastic cross section. ${ }^{13}$ Data at much closer energy intervals, shown as solid dots, were taken in a less time consuming manner by observing the relative differential cross section of He, correcting it with a smooth function to the theoretical shape, and applying the same correction to the signal from $\mathrm{CH}_{3} \mathrm{Cl}$, thus eliminating energy dependent electron optical variations. Following this, the cross section was then normalized in an absolute sense at a single energy. Good agreement was found between the two methods.
TABLE I. Energy dependence of the differential cross section for electrons elastically scattered from $\mathrm{CH}_{3} \mathrm{Cl}$, units of $10^{-16} \mathrm{~cm}^{2} / \mathrm{sr}$.

\begin{tabular}{crc}
\hline \hline Energy $(\mathrm{eV})$ & $30^{\circ}$ & $100^{\circ}$ \\
\hline 0.5 & 19.32 & 1.89 \\
0.8 & 12.25 & 1.43 \\
1.0 & 9.51 & 1.31 \\
1.5 & 7.02 & 1.38 \\
2.0 & 5.56 & 1.52 \\
2.5 & 4.87 & 1.64 \\
3.0 & 4.72 & 1.85 \\
3.2 & 4.53 & 1.97 \\
3.4 & 4.63 & 1.92 \\
3.6 & 4.83 & 1.93 \\
4.0 & 4.73 & 1.87 \\
4.5 & 4.70 & 1.79 \\
5.0 & 5.11 & 1.62 \\
5.2 & 5.14 & 1.54 \\
5.4 & 5.27 & 1.49 \\
5.6 & 5.39 & 1.44 \\
5.8 & 5.40 & 1.37 \\
6.0 & 5.50 & 1.34 \\
6.5 & 5.74 & 1.19 \\
7.0 & 5.86 & 1.16 \\
8.0 & 6.10 & 1.08 \\
9.0 & 6.57 & 1.05 \\
\hline \hline
\end{tabular}

\section{RESULTS}

\section{A. Elastic scattering: Energy dependence}

In Fig. 1 we show the differential elastic (vibrationally elastic) cross sections for scattering at $30^{\circ}$ and $100^{\circ}$ over an energy range from 0.5 to $9 \mathrm{eV}$. Note the considerable difference in scale between the two drawings. The data indicated with open circles were normalized to He at each energy. The error bar indicates the statistical spread in the data, approximately $6 \%$; that for the $30^{\circ}$ data is approximately the same size as the open circles. Systematic errors are estimated to be $5 \%$ or less. The solid line marked BDA, calculated using the Born dipole approximation, ${ }^{14}$ shows the contribution of scattering from the static electric dipole moment of $\mathrm{CH}_{3} \mathrm{Cl}$, given as $1.889 \mathrm{D}$ by Radzik and Smirnov. ${ }^{15}$ At the very lowest energies, differential elastic cross sections at both angles are dominated by the dipole contribution.

At $100^{\circ}$ a clear peak in the cross section occurs at $3.6 \mathrm{eV}$ with a full width at half-maximum of approximately $2.6 \mathrm{eV}$. This feature was first observed in the total scattering cross section ${ }^{16}$ using electron transmission spectroscopy ${ }^{17}$ (ETS) and attributed to the ${ }^{2} A_{1}$ ground state of the $\mathrm{CH}_{3} \mathrm{Cl}$ anion, formed by occupation of the $8 a_{1}$ antibonding $\mathrm{C}-\mathrm{Cl}$ molecular orbital. This assignment was supported by a number of calculations using various theoretical methods. ${ }^{16,18,19}$ The feature is not visible in the $30^{\circ}$ data because of the sharply rising contribution of the dipole scattering at decreasing energy and steadily increasing potential scattering, other than dipole, above the resonance. The differential cross section data at the open circles are listed in Table I for reference.

\section{B. Elastic scattering: Angular dependence}

Figure 2 illustrates the angular variation in the scattering, listed in Table II, at selected energies below, at, and 

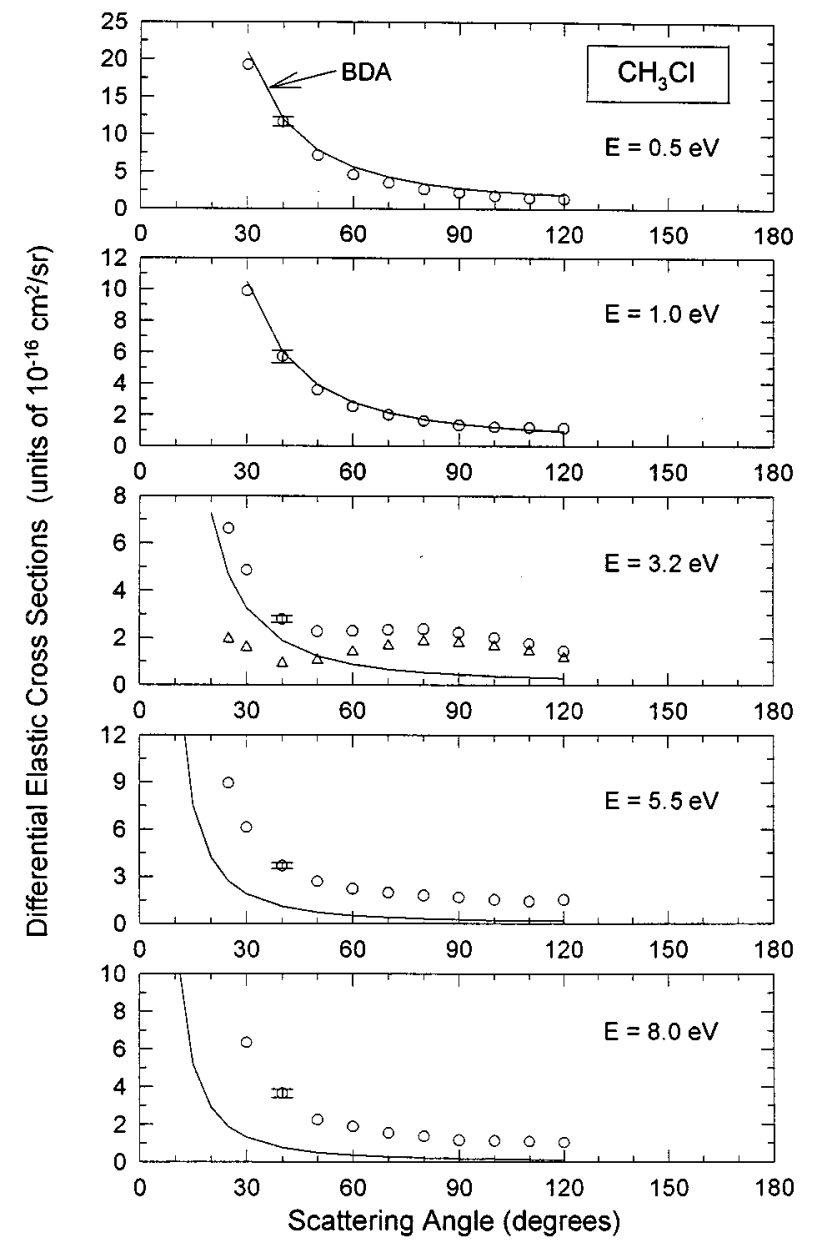

FIG. 2. The differential cross section for elastic scattering of electrons as a function of angle at $0.5,1.0,3.2,5.0$, and $8.0 \mathrm{eV}$. The solid line indicates the contribution from the Born dipole approximation. The open triangles in the data at $3.2 \mathrm{eV}$ show the result of subtracting the BDA contribution from the total.

above the ${ }^{2} A_{1}$ resonance. The solid line again indicates the calculated contribution from the Born dipole approximation. At 0.5 and $1.0 \mathrm{eV}$, well below the resonance, the dipole cross section accounts completely for the scattering. At $0.5 \mathrm{eV}$ our data fall consistently just below the dipole curve although the deviation is approximately the same size as the error bars. This may arise from the use of the point dipole approximation in the calculation rather than the physical distributed dipole. $^{20}$

At $3.2 \mathrm{eV}$, close to the top of the resonance, the scattering no longer declines monotonically with increasing angle but displays a maximum near $80^{\circ}$. After subtraction of the dipole contribution, the data, shown as open triangles, reveal a minimum at $40^{\circ}$.

\section{Electron energy loss spectra}

\section{Incident energy of $3.5 \mathrm{eV}$}

Figure 3 displays the vibrational energy loss data in $\mathrm{CH}_{3} \mathrm{Cl}$. For comparison, in Fig. 4 we also include results in
TABLE II. Angular distribution of the differential cross section for electrons elastically scattered from $\mathrm{CH}_{3} \mathrm{Cl}$ at selected energies, units of $10^{-16} \mathrm{~cm}^{2} / \mathrm{sr}$.

\begin{tabular}{crrrrr}
\hline \hline$\theta$ (degrees) & $0.5 \mathrm{eV}$ & $1.0 \mathrm{eV}$ & $3.2 \mathrm{eV}$ & $5.5 \mathrm{eV}$ & $8.0 \mathrm{eV}$ \\
\hline 25 & & & 6.63 & 8.93 & \\
30 & 19.24 & 9.88 & 4.86 & 6.12 & 6.34 \\
40 & 11.67 & 5.72 & 2.79 & 3.68 & 3.65 \\
50 & 7.18 & 3.59 & 2.27 & 2.67 & 2.24 \\
60 & 4.58 & 2.52 & 2.29 & 2.20 & 1.87 \\
70 & 3.50 & 2.00 & 2.34 & 1.97 & 1.53 \\
80 & 2.68 & 1.61 & 2.38 & 1.78 & 1.37 \\
90 & 2.19 & 1.34 & 2.22 & 1.66 & 1.15 \\
100 & 1.78 & 1.22 & 2.01 & 1.51 & 1.10 \\
110 & 1.47 & 1.17 & 1.77 & 1.41 & 1.08 \\
120 & 1.38 & 1.15 & 1.44 & 1.51 & 1.02 \\
\hline \hline
\end{tabular}

$\mathrm{CD}_{3} \mathrm{Cl}$ published earlier. ${ }^{1}$ These and the remaining energy loss spectra in Figs. 5 and 6 were collected at a scattering angle of $100^{\circ}$ to reduce the contributions from dipole allowed excitations. We note that all of the vibrational modes, which are listed by symmetry and class of deformation in Table III, are infrared and Raman active. ${ }^{21}$

The vibrational excitation that takes place near the peak of the ${ }^{2} A_{1}$ resonance, shown in Figs. 3 and 4, has been discussed earlier in our brief report. ${ }^{1}$ Qualitatively, the vibrational modes which are expected to be most strongly excited are those permitted by the symmetry of the ${ }^{2} A_{1}$ resonance, namely the $a_{1}$ modes, ${ }^{22}$ and which have strongly antibonding charge distributions. Thus, strongest excitation is expected for the $v_{3}^{\mathrm{C}-\mathrm{Cl}}\left(a_{1}\right)$ mode at $90.9 \mathrm{meV}$ and its overtones. Because of the smaller coefficients of the $8 a_{1}$ wave function elsewhere, excitation of the $v_{2}^{\mathrm{CH}_{3}}\left(a_{1}\right)$ and $v_{1}^{\mathrm{CH}}\left(a_{1}\right)$ modes will be considerably weaker. Figure 3 indeed shows that the fundamental of the $\mathrm{C}-\mathrm{Cl}$ stretching mode is the major excitation.

Because of the close proximity of the $v_{5}^{\mathrm{CH}_{3}}(e)$ mode to

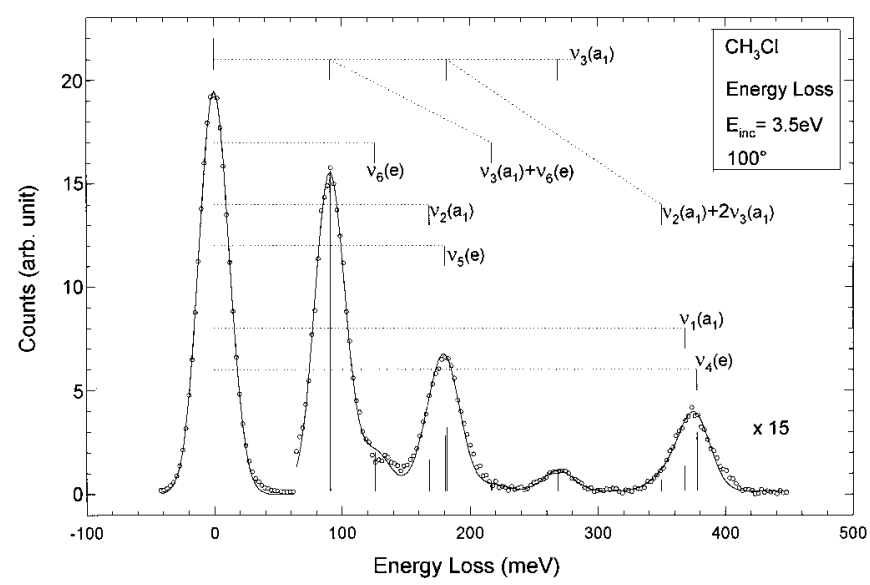

FIG. 3. Energy loss spectrum of $\mathrm{CH}_{3} \mathrm{Cl}$ at an incident electron energy of 3.5 $\mathrm{eV}$ and a scattering angle of $100^{\circ}$. The solid line shows a best fit to the data using the elastic peak as an instrumental function. The relative contributions of the vibrational levels are shown as sticks placed at the appropriate energies. 


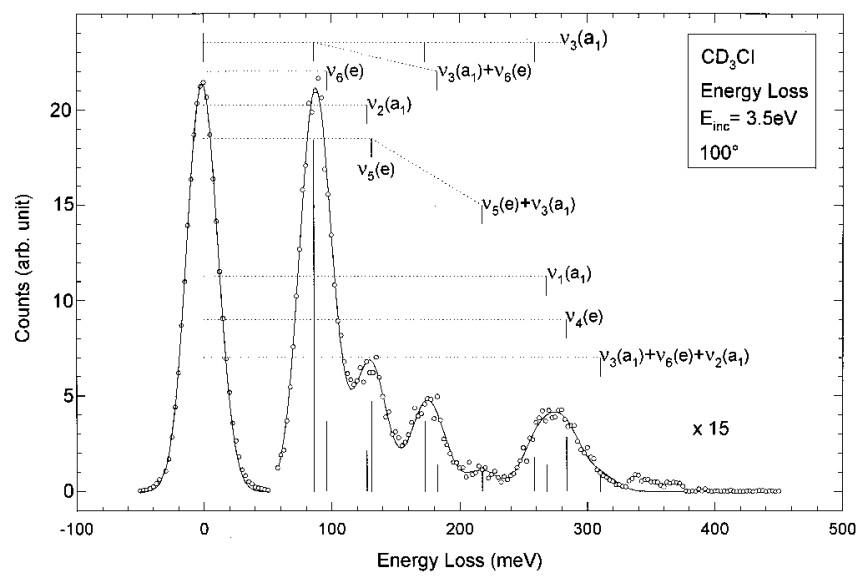

FIG. 4. Energy loss spectrum of $\mathrm{CD}_{3} \mathrm{Cl}$ as in Fig. 3.

$2 v_{3}^{\mathrm{C}-\mathrm{Cl}}\left(a_{1}\right)$ in $\mathrm{CH}_{3} \mathrm{Cl}$, it was not possible in Fig. 3 to confirm whether the first overtone of the $v_{3}^{\mathrm{C}-\mathrm{Cl}}\left(a_{1}\right)$ mode was present. Thus, data were taken in $\mathrm{CD}_{3} \mathrm{Cl}$ (MER isotopes) to split this near degeneracy. As Fig. 4 shows, in the deuterated compound it is possible to assign the relatively isolated $2 v_{3}$ overtone with no difficulty. Returning to Fig. 3, the next overtone, $3 v_{3}$, is clearly visible in $\mathrm{CH}_{3} \mathrm{Cl}$.

As we discussed earlier, ${ }^{1}$ the energy loss spectrum at 3.5 $\mathrm{eV}$ is made more complex because the ${ }^{2} A_{1}$ resonance is overlapped by the low energy tail of a second resonance. As observed in the vibrational excitation function of the $v_{4}(e)$ mode shown later in the paper, the higher feature peaks near $5.5 \mathrm{eV}$. We have attributed this resonance to the ${ }^{2} E$ temporary anion state formed by occupation of the $4 e$ molecular orbital. ${ }^{1}$ The symmetry of this state permits excitation of both $e$ and $a_{1}$ modes. ${ }^{22}$ Excitation of the $v_{3}^{\mathrm{C}-\mathrm{Cl}}\left(a_{1}\right)$ mode should be very weak through this resonance because of the unfavorable charge distribution, but more substantial excitation of certain of the $v_{2}^{\mathrm{CH}_{3}}\left(a_{1}\right), v_{1}^{\mathrm{CH}}\left(a_{1}\right), v_{6}(e), v_{5}^{\mathrm{CH}_{3}}(e)$, and

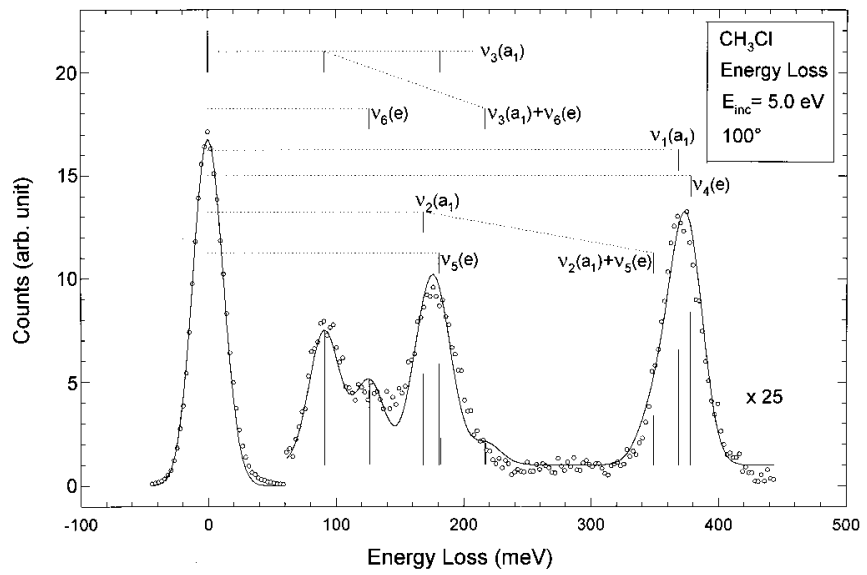

FIG. 5. Energy loss spectrum of $\mathrm{CH}_{3} \mathrm{Cl}$ at an incident energy of $5.0 \mathrm{eV}$ and a scattering angle of $100^{\circ}$.

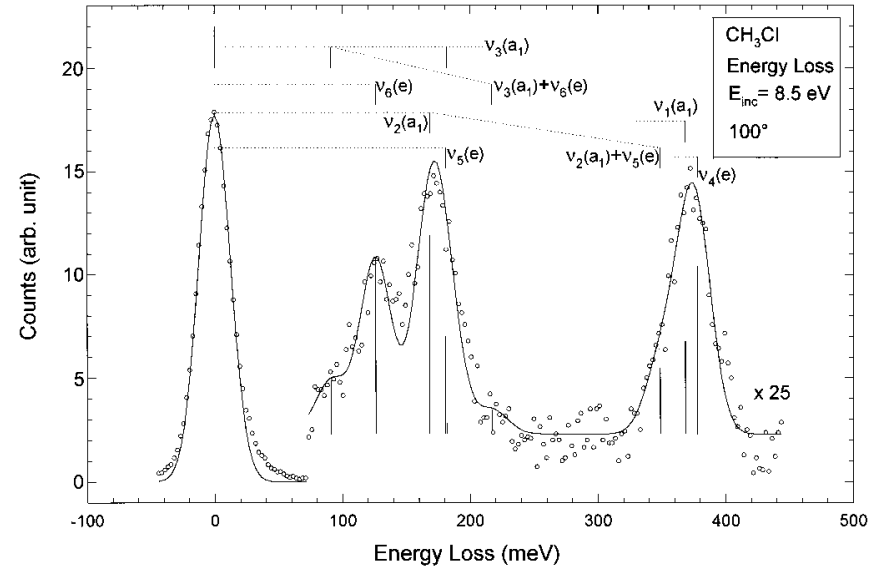

FIG. 6. Energy loss spectrum of $\mathrm{CH}_{3} \mathrm{Cl}$ at $8.5 \mathrm{eV}$ and a scattering angle of $100^{\circ}$.

$v_{4}^{\mathrm{CH}}(e)$ modes may be possible depending on the detailed shape of the charge density.

In an attempt to extract more quantitative data from the loss spectra, we have carried out a fit to the data in Figs. 3 and 4 in a straightforward manner. An instrument function was obtained by fitting a Gaussian profile to the measured elastic peak. Using the known energies of the fundamentals and several intercombination modes, a least-squares fit to determine the weighting of each important peak was obtained. The energy of $3 v_{3}$ was allowed to vary slightly for best fit and was found to be $268.9 \mathrm{meV}$, reflecting a reasonable anharmonicity of about $4 \%$. The solid line passing through the data points indicates the overall agreement. The stick figures indicate the relative contributions from the various losses. The weightings relative to the size of the fundamental $\mathrm{C}-\mathrm{Cl}$ stretching vibration for $\mathrm{CH}_{3} \mathrm{Cl}$ are listed in Table IV, and those for $\mathrm{CD}_{3} \mathrm{Cl}$ in Table V.

Because of the close proximity of $2 v_{3}\left(a_{1}\right)$ to $v_{5}(e)$ in $\mathrm{CH}_{3} \mathrm{Cl}$ alluded to earlier, attempts to include them separately in the fitting program were not successful. Instead, the data in $\mathrm{CD}_{3} \mathrm{Cl}$, in which these levels are well isolated, were fit first and the weight of $2 v_{3}$ relative to $v_{3}$ determined. We next assumed that the same ratio would apply in $\mathrm{CH}_{3} \mathrm{Cl}$. Enforcing this, we extracted the weighting for the $v_{5}$ level. We return to a discussion of the relative weights later in the paper.

TABLE III. The vibrational modes of $\mathrm{CH}_{3} \mathrm{Cl}$ and $\mathrm{CD}_{3} \mathrm{Cl}$, their symmetries, energies, and the types of deformation (see Ref. 21).

\begin{tabular}{clcc}
\hline \hline $\begin{array}{c}\text { Mode } \\
\text { (symmetry) }\end{array}$ & Deformation & $\begin{array}{c}\mathrm{CH}_{3} \mathrm{Cl} \\
\text { energy (meV) }\end{array}$ & $\begin{array}{c}\mathrm{CD}_{3} \mathrm{Cl} \\
\text { energy }(\mathrm{meV})\end{array}$ \\
\hline$\nu_{3}\left(a_{1}\right)$ & $\mathrm{C}-\mathrm{Cl}$ stretch & 90.9 & 86.3 \\
$\nu_{6}(e)$ & $\mathrm{CH}_{3}$ rock & 126.1 & 96.3 \\
$\nu_{2}\left(a_{1}\right)$ & $\mathrm{CH}_{3} s$ deform & 168.3 & 127.8 \\
$\nu_{5}(e)$ & $\mathrm{CH}_{3} d$ deform & 180.7 & 131.4 \\
$\nu_{1}\left(a_{1}\right)$ & $\mathrm{CH} s$ stretch & 368.4 & 268.4 \\
$\nu_{4}(e)$ & $\mathrm{CH} d$ stretch & 377.8 & 283.9 \\
\hline \hline
\end{tabular}


TABLE IV. Decomposition of the energy loss spectrum in $\mathrm{CH}_{3} \mathrm{Cl}$ at $E_{\text {inc }}=3.5 \mathrm{eV}$.

\begin{tabular}{lcc}
\hline \hline $\begin{array}{c}\text { Mode } \\
\text { (symmetry) }\end{array}$ & $\begin{array}{c}\mathrm{CH}_{3} \mathrm{Cl} \\
\text { energy }(\mathrm{meV})\end{array}$ & $\begin{array}{c}\text { Normalized } \\
\text { strength }\end{array}$ \\
\hline & Fundamentals & \\
$\nu_{3}\left(a_{1}\right)$ & 90.9 & 1.00 \\
$\nu_{6}(e)$ & 126.1 & 0.12 \\
$\nu_{2}\left(a_{1}\right)$ & 168.3 & 0.10 \\
$\nu_{5}(e)$ & 180.7 & $0.17^{\mathrm{a}}$ \\
$\nu_{1}\left(a_{1}\right)$ & 368.4 & 0.08 \\
$\nu_{4}(e)$ & 377.8 & 0.18 \\
& & \\
$2 \nu_{3}\left(a_{1}\right)$ & Overtones & $0.20^{\mathrm{a}}$ \\
$3 \nu_{3}\left(a_{1}\right)$ & 181.8 & 0.06 \\
& 268.9 & \\
$\nu_{3}\left(a_{1}\right)+\nu_{6}(e)$ & Mixed & 0.02 \\
$\nu_{2}\left(a_{1}\right)+2 \nu_{3}\left(a_{1}\right)$ & 217.0 & 0.03 \\
\hline \hline
\end{tabular}

${ }^{\mathrm{a}}$ See text.

\section{Incident energies of 5.0 and $8.5 \mathrm{eV}$}

The fittings in Figs. 3 and 4 indicate that although the dominant excitation is to a mode of $a_{1}$ symmetry, there is a sizeable contribution at $3.5 \mathrm{eV}$ from $e$-type modes made up primarily of $\mathrm{CH}_{3}$ deformations and stretches. Energy loss spectra taken at 5.0 and $8.5 \mathrm{eV}$ are presented in Figs. 5 and 6. Although the signal to noise is not as good as in the loss data at $3.5 \mathrm{eV}$, we have fit the results satisfactorily using almost the same set of frequencies as in Figs. 3 and 4. The peak intensities relative to that for $v_{3}$ are given in Table VI. We have again assumed that the $2 v_{3}$ overtone has the same size relative to its fundamental as before.

In moving from Fig. 3 to Figs. 5 and 6, the most significant trend is the rapid decline of excitation of the $\mathrm{C}-\mathrm{Cl}$ vibrations. At the intermediate energy of $5.0 \mathrm{eV}, v_{3}$ is still excited, however, it is now comparable in size to the other fundamental modes. At $8.5 \mathrm{eV}, v_{3}$ is barely detectable and considerably smaller than the others.

\section{Vibrational excitation cross sections}

\section{Energy dependence}

The changing ratios of $a_{1}$ and $e$ vibrational modes as a function of electron energy can be greatly clarified by measurement of the differential cross sections for vibrational excitation of $v_{3}^{\mathrm{C}-\mathrm{Cl}}\left(a_{1}\right)$ and the loss peak at $\approx 375 \mathrm{meV}$ over the range from 1.5 to $10 \mathrm{eV}$, shown in Figs. 7 and 8 respectively. The scattering angle was $100^{\circ}$ in each case. The data shown in open circles were determined by reference to the elastic peak at each impact energy and use of the absolute calibration of the elastic cross section in Fig. 1 and are listed in Table VII. The data shown as solid dots were acquired as in Fig. 1, using the previously determined correction for electron optical variations, and normalizing only at a single impact energy. It should be noted that although $v_{4}^{\mathrm{CH}}(e)$ is the largest contributor to the loss peak at $375 \mathrm{meV}$, there remains a substantial contribution from $v_{1}^{\mathrm{CH}}\left(a_{1}\right)$, which lies below $v_{4}$ by only $9.4 \mathrm{meV}$. The cross section is more appropriately
TABLE V. Decomposition of the energy loss spectrum in $\mathrm{CD}_{3} \mathrm{Cl}$ at $E_{\text {inc }}=3.5$ $\mathrm{eV}$.

\begin{tabular}{lcc}
\hline \hline $\begin{array}{c}\text { Mode } \\
\text { (symmetry) }\end{array}$ & $\begin{array}{c}\mathrm{CD}_{3} \mathrm{Cl} \\
\text { energy }(\mathrm{meV})\end{array}$ & $\begin{array}{c}\text { Normalized } \\
\text { Strength }\end{array}$ \\
\hline \multicolumn{3}{c}{ Fundamentals } \\
$\nu_{3}\left(a_{1}\right)$ & 86.3 & 1.00 \\
$\nu_{6}(e)$ & 96.3 & 0.20 \\
$\nu_{2}\left(a_{1}\right)$ & 127.8 & 0.12 \\
$\nu_{5}(e)$ & 131.4 & 0.25 \\
$\nu_{1}\left(a_{1}\right)$ & 268.4 & 0.08 \\
$\nu_{4}(e)$ & 283.9 & 0.15 \\
& & \\
$2 \nu_{3}\left(a_{1}\right)$ & Overtones & 0.20 \\
$3 \nu_{3}\left(a_{1}\right)$ & 172.6 & 0.10 \\
& 259.0 & \\
$\nu_{3}\left(a_{1}\right)+\nu_{6}(e)$ & Mixed & 0.08 \\
$\nu_{3}\left(a_{1}\right)+\nu_{5}(e)$ & 182.6 & 0.06 \\
$\nu_{3}\left(a_{1}\right)+\nu_{6}(e)+\nu_{2}\left(a_{1}\right)$ & 217.7 & 0.05 \\
\hline \hline
\end{tabular}

attributed to a mixture of the two. The solid line passing through the data in Figs. 7 and 8 will be discussed later.

The different energy dependences for the $\mathrm{C}-\mathrm{Cl}$ and $\mathrm{C}-\mathrm{H}$ modes bear out the interpretation of $\mathrm{a}^{2} A_{1}$ anion state peaking near $3.5 \mathrm{eV}$ and $\mathrm{a}^{2} E$ state near $5.5 \mathrm{eV}$. Because of the breadth of the latter, there is appreciable excitation of certain of the $e$ modes even at $3.5 \mathrm{eV}$ as seen in Figs. 3 and 4. We note that the $v_{3}$ excitation taking place through the ${ }^{2} A_{1}$ resonance is maximum near $3.2 \mathrm{eV}$, somewhat below the energy of the maximum in the elastic channel. This likely reflects the difference in the Franck-Condon factors.

Calculations of the lowest empty orbitals of methyl chloride $^{16}$ indicate the existence of a second orbital of $a_{1}$ symmetry above the $4 e$ orbital. This resonance is not seen in our data, although the possibility cannot be ruled out that it contributes to the broad peak in the excitation of $v_{4}(e) / v_{1}\left(a_{1}\right)$ shown in Fig. 8. In a related molecule, $\mathrm{CF}_{3} \mathrm{Cl}$,

TABLE VI. Decomposition of the energy loss spectra in $\mathrm{CH}_{3} \mathrm{Cl}$ at $E_{\text {inc }}=5.0$ and $8.5 \mathrm{eV}$.

\begin{tabular}{lccc}
\hline \hline $\begin{array}{c}\text { Mode } \\
(\text { symmetry })\end{array}$ & $\begin{array}{c}\mathrm{CH}_{3} \mathrm{Cl} \\
\text { energy }(\mathrm{meV})\end{array}$ & $\begin{array}{c}\text { Normalized strength } \\
\left(E_{\text {inc }}=5.0 \mathrm{eV}\right)\end{array}$ & $\begin{array}{c}\text { Normalized strength } \\
\left(E_{\text {inc }}=8.5 \mathrm{eV}\right)\end{array}$ \\
\hline & \multicolumn{3}{c}{ Fundamentals } \\
$\nu_{3}\left(a_{1}\right)$ & 90.9 & 1.00 & 1.00 \\
$\nu_{6}(e)$ & 126.1 & 0.63 & 3.31 \\
$\nu_{2}\left(a_{1}\right)$ & 168.3 & 0.68 & 3.74 \\
$\nu_{5}(e)$ & 180.7 & 0.75 & 1.83 \\
$\nu_{1}\left(a_{1}\right)$ & 368.4 & 0.86 & 1.74 \\
$\nu_{4}(e)$ & 377.8 & 1.15 & 3.16 \\
& & & \\
$2 \nu_{3}\left(a_{1}\right)$ & 181.8 & $0.20^{\mathrm{a}}$ & $0.20^{\mathrm{a}}$ \\
$3 \nu_{3}\left(a_{1}\right)$ & 268.9 & 0.00 & 0.00 \\
& & & \\
$\nu_{3}\left(a_{1}\right)+\nu_{6}(e)$ & 217.0 & 0.16 & 0.46 \\
$\nu_{2}\left(a_{1}\right)+\nu_{5}(e)$ & 349.0 & 0.37 & 1.24 \\
\hline \hline
\end{tabular}

${ }^{\mathrm{a}}$ See text. 


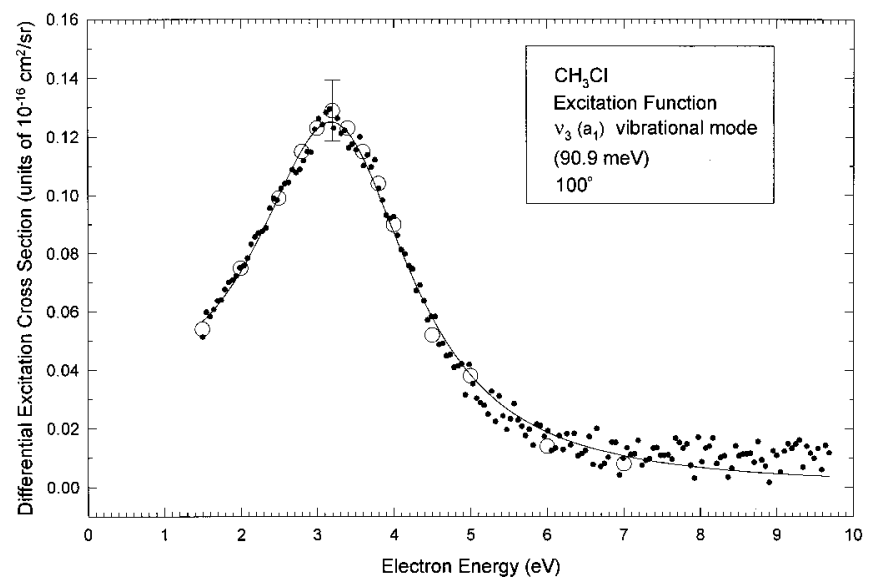

FIG. 7. Differential cross section for the excitation of $v_{3}\left(a_{1}\right)$ as a function of energy in $\mathrm{CH}_{3} \mathrm{Cl}$ at a scattering angle of $100^{\circ}$. The solid line shows an empirical fit to the data using a single-level Breit-Wigner resonance formula with a width function linearly dependent on energy.

Mann and Linder ${ }^{23}$ observed the second ${ }^{2} A_{1}$ resonance at an energy of $8.5 \mathrm{eV}$ in the $v_{2}\left(a_{1}\right)$ excitation function.

\section{Angular dependence}

The dependence on scattering angle of the differential cross sections for excitation of $v_{3}^{\mathrm{C}-\mathrm{Cl}}\left(a_{1}\right)$ and $v_{4}^{\mathrm{CH}}(e)$ plus the smaller admixture of $v_{1}^{\mathrm{CH}}\left(a_{1}\right)$ is shown in Fig. 9 and listed in Table VIII. The data for $v_{3}$ reveal a shallow minimum near $40^{\circ}$ and a maximum near $80^{\circ}$ as also observed in the elastic scattering at $3.2 \mathrm{eV}$ in Fig. 2. Two calculations of the angular dependence for excitation of the $v_{3}$ mode at $3.2 \mathrm{eV}$ have been carried out $^{2,24}$ and compared previously with our data. ${ }^{24} \mathrm{Un}$ fortunately, neither model contains all the necessary physics to provide a valid comparison with experiment. The calculations by Gallup ${ }^{2}$ do not take account of the influence of the permanent dipole moment of the molecule. The calculations of Fabrikant, ${ }^{24}$ on the other hand, include the permanent dipole moment but do not allow the full range of spherical harmonics required to reflect the tunneling out of the $8 a_{1}$ orbital.

The angular distribution for excitation of $v_{4}$ through the ${ }^{2} E$ resonance has been calculated by Gallup and compared with our data previously. ${ }^{2}$ Again the theory did not include effects due to the permanent dipole moment.

\section{THEORETICAL COMPARISONS}

The new measurements described in this article allow us to greatly improve our knowledge of the resonance parameters, particularly those of the $e$ resonance. We have therefore recalculated the strengths of the energy loss peaks for vibrational excitation, following the theory given before by Gallup. ${ }^{2}$ In this theory, a mixed semiempirical $a b$ initio approach was taken that requires the determination from experiment of the electronic properties of the resonance such as energy and width. The other quantities that go into the theory have not been changed from those used in the previous article, to which we direct the reader for details.

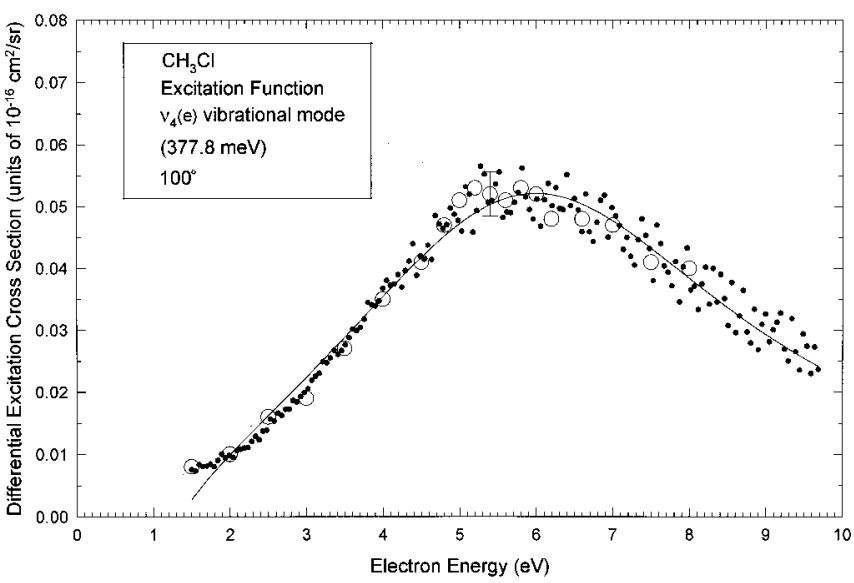

FIG. 8. Differential cross section for the excitation of the $375 \mathrm{meV}$ loss peak, primarily $v_{4}(e)$, as a function of energy at a scattering angle of $100^{\circ}$. It should be noted that the cross section also contains a contribution from $v_{1}\left(a_{1}\right)$.

Isolated resonances are frequently described by the Breit-Wigner single level formula. ${ }^{25}$ This model assumes that the width of the resonance is small compared to its energy. Under these circumstances it is sensible to speak of the width as if it is constant and, in addition, to ignore the $1 / E$ factor, always present in the cross section expression. Shape resonances more generally, and the resonances in $\mathrm{CH}_{3} \mathrm{Cl}$ in particular, do not come close to satisfying such conditions. On the other hand, we resist the temptation to use too many parameters in empirical fitting; otherwise, the results become unconvincing. As a compromise between the two extremes, we have used

TABLE VII. Energy dependence of the differential cross section for electrons exciting the $v_{3}\left(a_{1}\right)$ and $v_{4}(e)$ modes at $100^{\circ}$, units of $10^{-16} \mathrm{~cm}^{2} / \mathrm{sr}$.

\begin{tabular}{cccc}
\hline \hline Energy $(\mathrm{eV})$ & $\nu_{3}\left(a_{1}\right)$ mode & Energy $(\mathrm{eV})$ & $\nu_{4}(e)$ mode $^{\mathrm{a}}$ \\
\hline 1.5 & 0.054 & 1.5 & 0.008 \\
2.0 & 0.075 & 2.0 & 0.010 \\
2.5 & 0.099 & 2.5 & 0.016 \\
3.0 & 0.115 & 3.0 & 0.019 \\
2.8 & 0.123 & 3.5 & 0.027 \\
3.2 & 0.129 & 4.0 & 0.035 \\
3.4 & 0.123 & 4.5 & 0.041 \\
3.6 & 0.115 & 4.8 & 0.047 \\
3.8 & 0.104 & 5.0 & 0.051 \\
4.0 & 0.090 & 5.2 & 0.053 \\
4.5 & 0.052 & 5.4 & 0.052 \\
5.0 & 0.038 & 5.6 & 0.051 \\
6.0 & 0.014 & 5.8 & 0.053 \\
7.0 & 0.008 & 6.0 & 0.052 \\
& & 6.2 & 0.048 \\
& & 6.6 & 0.048 \\
& & 7.0 & 0.047 \\
& & 7.5 & 0.041 \\
\hline \hline
\end{tabular}

${ }^{\mathrm{a} C}$ Contains an admixture of $\nu_{1}\left(a_{1}\right)$. 

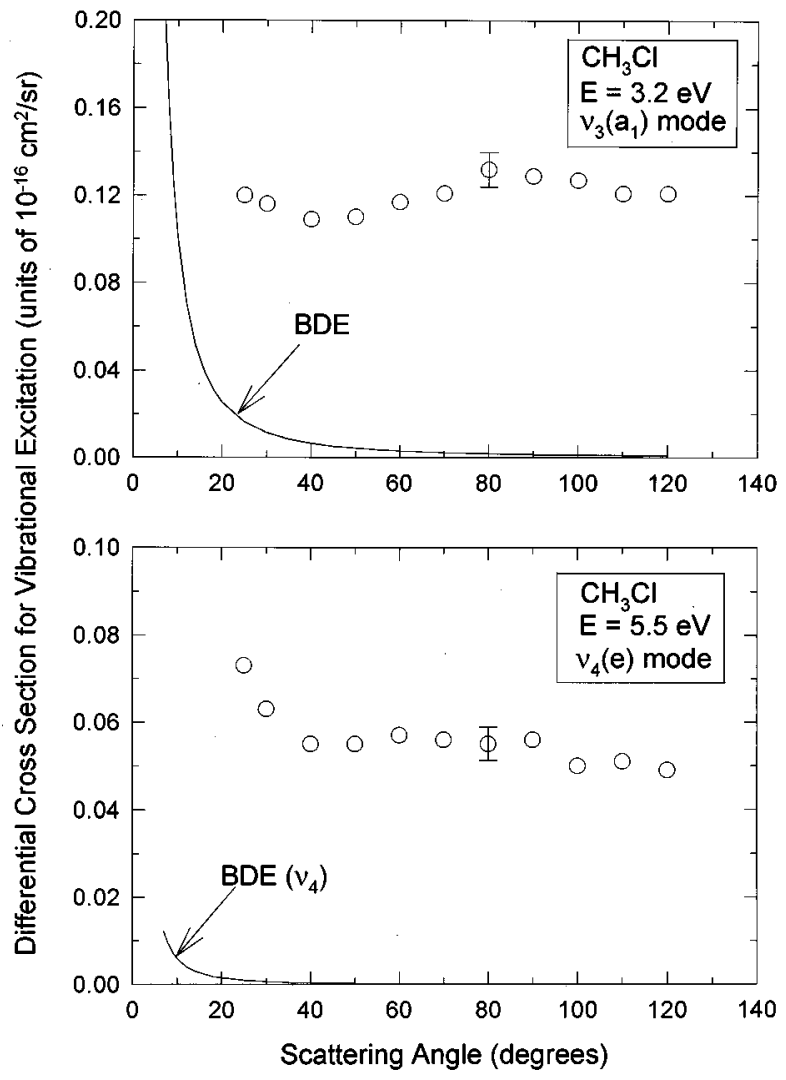

FIG. 9. Differential cross section for vibrational excitation of $v_{3}\left(a_{1}\right)$ at 3.2 $\mathrm{eV}$ (upper panel) and $v_{4}(e)$ at $5.5 \mathrm{eV}$ (lower panel) as a function of scattering angle. The solid line marked BDE indicates the contribution from Born dipole excitation.

$$
P(E)=\frac{A \Gamma(E) / 2}{E\left[\left(E-E_{0}\right)^{2}+\Gamma(E)^{2} / 4\right]},
$$

to represent the profile of the resonances in $\mathrm{CH}_{3} \mathrm{Cl}$ that we consider. In this, we let $\Gamma(E)$ be represented by a simple linear expression,

$$
\Gamma(E)=\Gamma_{0}+\Gamma_{1}\left(E-E_{0}\right) .
$$

Least-square fits of this expression to the excitation functions for $\nu_{3}$ through the ${ }^{2} A_{1}$ resonance and for $\nu_{4}$ by the ${ }^{2} E$ resonance are shown by the solid lines in Figs. 7 and 8. The four parameters $A, E_{0}, \Gamma_{0}$, and $\Gamma_{1}$ were varied to obtain the agreement. The parameters for the ${ }^{2} A_{1}$ resonance were not changed significantly from those used in Ref. 2 . The values for the ${ }^{2} E$ resonance are significantly different, however, and the inclusion of these in the calculation of the excitation function at $3.5 \mathrm{eV}$ greatly improves the overall agreement of the theory with experiment. The resonance parameters in or$\operatorname{der} A, E_{0}, \Gamma_{0}$, and $\Gamma_{1}$ are $0.549,3.601 \mathrm{eV}, 2.705 \mathrm{eV}$, and 0.549 for the ${ }^{2} A_{1}$ resonance, and $1.560,8.261 \mathrm{eV}, 10.521 \mathrm{eV}$, and 1.521 for the ${ }^{2} E$ resonance. In spite of the quality of the fit to the experimental data, an expression of this sort must be used with care. Our approximation for $\Gamma(E)$ is not nonnegative for all $E$ as the correct expression must be. In addition, our empirical function is not realistic near $E=0$, since $\Gamma(E)$ will obey no sensible threshold law. As pointed out by
TABLE VIII. Angular distributions of differential cross section for electrons exciting the $v_{3}\left(a_{1}\right)$ and $v_{4}(e)$ modes at selected energies, units of $10^{-16}$ $\mathrm{cm}^{2} / \mathrm{sr}$.

\begin{tabular}{ccc}
\hline \hline & $\begin{array}{c}\nu_{3}\left(a_{1}\right) \text { mode } \\
3.2 \mathrm{eV}\end{array}$ & $\begin{array}{c}\nu_{4}(e) \text { mode }^{\mathrm{a}} \\
5.5 \mathrm{eV}\end{array}$ \\
\hline 25 & 0.120 & 0.073 \\
30 & 0.116 & 0.063 \\
40 & 0.109 & 0.055 \\
50 & 0.110 & 0.055 \\
60 & 0.117 & 0.057 \\
70 & 0.121 & 0.056 \\
80 & 0.132 & 0.055 \\
90 & 0.129 & 0.056 \\
100 & 0.127 & 0.050 \\
110 & 0.121 & 0.051 \\
120 & 0.121 & 0.049 \\
\hline \hline
\end{tabular}

${ }^{\mathrm{a}}$ Contains an admixture of $\nu_{1}\left(a_{1}\right)$.

Domcke $^{26}$ in the $a b$ initio expression, there is an energy shift term $\Delta(E)$ related to $\Gamma(E)$ by an integral. We do not determine a large enough range of $\Gamma(E)$ to obtain the $\Delta(E)$ function, and so we omit it. As can be seen, the parameters we arrive at yield positive values for $\Gamma(E)$ in the range of the experimental data.

These results show that the location of the ${ }^{2} E$ resonance is, in fact, well above the peak in the vibrational excitation cross section, and its influence on excitation at $3.5 \mathrm{eV}$ is smaller than realized in the earlier calculation. ${ }^{2}$ With the new parameters the relative strengths for excitation of the vibrational modes of $\mathrm{CH}_{3} \mathrm{Cl}$ excited at $3.5 \mathrm{eV}$ have been recomputed using the method of Ref. 2. A list of the strengths normalized to that of the $v_{3}$ fundamental is given in Table IX and compared with the experimentally extracted strengths. The theoretical listing includes only those losses whose nor-

TABLE IX. Normalized strength of vibrational modes of $\mathrm{CH}_{3} \mathrm{Cl}$ excited at

\begin{tabular}{|c|c|c|c|}
\hline $\begin{array}{c}\text { Mode } \\
\text { (symmetry) }\end{array}$ & $\begin{array}{c}\mathrm{CH}_{3} \mathrm{Cl} \\
\text { energy }(\mathrm{meV})\end{array}$ & $\begin{array}{l}\text { Normalized } \\
\text { strength } \\
\text { (experiment) }\end{array}$ & $\begin{array}{l}\text { Normalized } \\
\text { strength } \\
\text { (theory) }\end{array}$ \\
\hline & \multicolumn{2}{|c|}{ Fundamentals } & \\
\hline$\nu_{3}\left(a_{1}\right)$ & 90.9 & 1.00 & 1.0000 \\
\hline$\nu_{6}(e)$ & 126.1 & 0.12 & 0.0081 \\
\hline$\nu_{2}\left(a_{1}\right)$ & 168.3 & 0.10 & 0.0313 \\
\hline$\nu_{5}(e)$ & 180.7 & $0.17^{\mathrm{a}}$ & 0.1200 \\
\hline$\nu_{1}\left(a_{1}\right)$ & 368.4 & 0.08 & 0.0594 \\
\hline \multirow[t]{2}{*}{$\nu_{4}(e)$} & 377.8 & 0.18 & 0.2206 \\
\hline & \multicolumn{2}{|c|}{ Overtones } & \\
\hline $2 \nu_{3}\left(a_{1}\right)$ & 181.8 & $0.20^{\mathrm{a}}$ & 0.2196 \\
\hline \multirow[t]{2}{*}{$3 \nu_{3}\left(a_{1}\right)$} & 268.9 & 0.06 & 0.0814 \\
\hline & \multicolumn{2}{|c|}{ Mixed } & \\
\hline$\nu_{3}\left(a_{1}\right)+\nu_{6}(e)$ & 217.0 & 0.02 & 0.0009 \\
\hline$\nu_{2}\left(a_{1}\right)+\nu_{3}\left(a_{1}\right)$ & 259.2 & & 0.0143 \\
\hline$\nu_{3}\left(a_{1}\right)+\nu_{5}(e)$ & 271.6 & & 0.0135 \\
\hline$\nu_{2}\left(a_{1}\right)+2 \nu_{3}\left(a_{1}\right)$ & 350.1 & 0.03 & 0.0076 \\
\hline $2 \nu_{5}(e)$ & 361.4 & & 0.0038 \\
\hline $2 \nu_{3}\left(a_{1}\right)+\nu_{5}(e)$ & 362.5 & & 0.0019 \\
\hline
\end{tabular}
$E_{\text {inc }}=3.5 \mathrm{eV}$.

${ }^{\mathrm{a}}$ See text. 


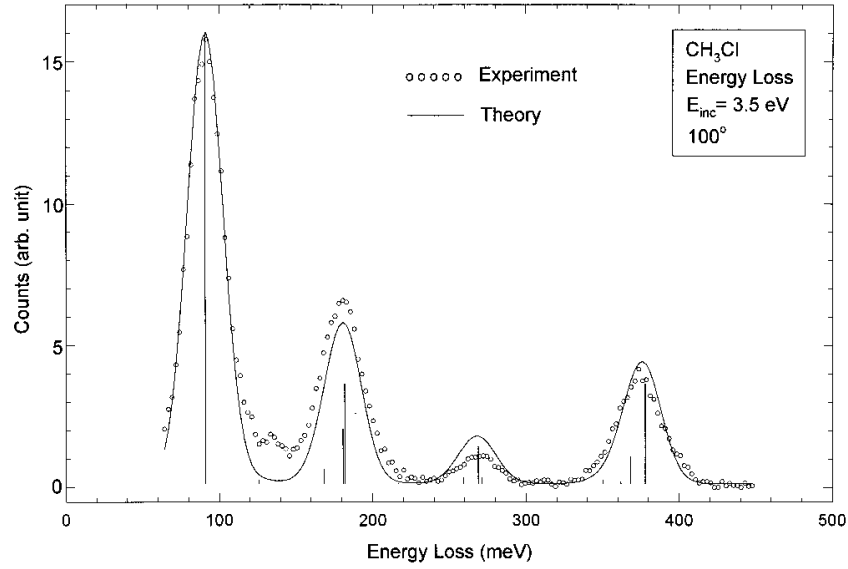

FIG. 10. Energy loss spectrum at an incident energy of $3.5 \mathrm{eV}$ and a scattering angle of $100^{\circ}$. The solid line shows a theoretical spectrum computed using the relative weights determined by the method of Ref. 2 and the known instrument function. Theory and experiment are normalized at the peak of the $v_{3}$ mode.

malized strengths are 0.0009 or larger. A theoretical energy loss spectrum at $3.5 \mathrm{eV}$ is shown in Fig. 10 using the tabulated strengths broadened by the instrumental function, along with the experimental data.

As judged by the theoretical loss spectrum in Fig. 10, the "lowest order" model ${ }^{2}$ gives a good account of the major loss peaks. The listing in Table IX indicates that the fundamental modes and $v_{3}$ overtones, with the exception of $v_{6}(e)$ and $v_{2}\left(a_{1}\right)$, agree with experiment within $35 \%$. The most serious difference lies in $v_{6}$, the $\mathrm{CH}_{3}$ rocking mode, which is about 15 times smaller in the calculation than in the experiment. However, closer examination of Fig. 3 reveals that the data are somewhat noisier and the quality of the fit in the region of $v_{6}(e)$ is not as good as elsewhere. Excitation to $v_{2}\left(a_{1}\right)$, the $\mathrm{CH}_{3} s$ deformation, is calculated to be 3.3 times smaller than measured. We have no arguments at present to suggest that these modes should be less accurately represented in the calculation.

\section{INTEGRATED CROSS SECTIONS}

Although we have reservations about the accuracy with which differential cross section data can be extrapolated to $0^{\circ}$ and $180^{\circ}$, it is useful to comment on the total cross sections derived from the integration of certain of our data.

The dominant energy loss at the ${ }^{2} A_{1}$ resonance at $3.2 \mathrm{eV}$ is to the $v_{3} \mathrm{C}-\mathrm{Cl}$ vibrational mode. The data in Fig. 9, taken from $25^{\circ}$ to $120^{\circ}$, indicate a relatively small variation with respect to angle. Ignoring the Born dipole contribution for the moment, and assuming that an average cross section of $0.12 \times 10^{-16} \mathrm{~cm}^{2} / \mathrm{sr}$ holds at all angles, we find a total resonant excitation cross section for this mode of $1.51 \times 10^{-16}$ $\mathrm{cm}^{2}$. The total direct excitation through the transition dipole is calculated to contribute only an additional $8.68 \times 10^{-18}$ $\mathrm{cm}^{2}$.

Using the relative strengths, listed in Table IX, of the other vibrational modes excited through the ${ }^{2} A_{1}$ resonance, we estimate the total inelastic scattering cross section due to vibrational excitation to be $2.96 \times 10^{-16} \mathrm{~cm}^{2}$. This excludes the much smaller dipole excitation.

Turning to the differential elastic scattering shown in Fig. 2, we note again that at impact energies of 0.5 and 1.0 $\mathrm{eV}$ there is excellent agreement between the measured data and the calculated cross section using the Born dipole approximation. Whereas, it is risky to extrapolate the experimental data to angles below $30^{\circ}$, it is straightforward to do the integration using the theoretical expression. The calculation was carried out using the dipole term for rotational excitation of symmetric top molecules given by Itikawa. ${ }^{27} \mathrm{Ad}-$ ditional details and a further exploration of this subject will be presented elsewhere. ${ }^{28}$

Because electron beam measurements of total scattering cross sections are always limited by the finite angle for rejection of electrons scattered near the forward direction, it is essential to limit the range of angular integration to make a comparison. The recent measurements of $\mathrm{CH}_{3} \mathrm{Cl}$ by Krzysztofowicz and Szmytkowski ${ }^{29}$ specify a detector solid angle of $1 \mathrm{msr}$. For this case, the calculated cross sections at 0.5 and $1.0 \mathrm{eV}$ are found to be 1.47 and $0.74 \times 10^{-14} \mathrm{~cm}^{2}$, respectively. The measured values reported ${ }^{29}$ at these energies are 0.44 and $0.34 \times 10^{-14} \mathrm{~cm}^{2}$, and the calculated cross sections are well outside of the listed experimental uncertainties. The measurements of Benitez et al. ${ }^{30}$ and Wan et al., ${ }^{31}$ using a magnetically collimated electron beam, lie even lower. Carrying out the integration down to 4 msr yields cross sections of 1.26 and $0.63 \times 10^{-14} \mathrm{~cm}^{2}$ at 0.5 and $1.0 \mathrm{eV}$, respectively, still well above the experimental values.

Because the scattering in $\mathrm{CH}_{3} \mathrm{Cl}$ is so clearly dominated by dipole effects at these low energies, and pressure measurements are relatively easy in comparison to molecules with similar dipole moments such as $\mathrm{H}_{2} \mathrm{O}$, this compound appears to be a good choice for further attempts to sort out the systematic errors in measurements of total scattering in polar molecules.

\section{ACKNOWLEDGMENTS}

This work was supported by the National Science Foundation, Grant No. CHE-9300941. Thanks are also due for many useful conversations with Professors K. D. Jordan and Ilya Fabrikant.

${ }^{1}$ X. Shi, T. M. Stephen, and P. D. Burrow, J. Chem. Phys. 96, 4037 (1992).

${ }^{2}$ G. A. Gallup, J. Phys. B 26, 759 (1993).

${ }^{3}$ J. Comer and F. H. Read, J. Phys. B 4, 368 (1971).

${ }^{4}$ M. J. W. Boness and G. J. Schulz, Phys. Rev. A 9, 1969 (1974).

${ }^{5}$ X. Shi and P. D. Burrow, J. Phys. B 25, 4273 (1992).

${ }^{6}$ X. Shi, T. M. Stephen, and P. D. Burrow, J. Phys. B 26, 121 (1993).

${ }^{7}$ S. K. Srivastava, A. Chutjian, and S. Trajmar, J. Chem. Phys. 63, 2659 (1975), J. C. Nickel, P. W. Zetner, G. Shen, and S. Trajmar, J. Phys. E 22, 730 (1989).

${ }^{8}$ H. P. Saha, Phys. Rev. Lett. 65, 2003 (1990).

${ }^{9}$ T. F. O'Malley and R. W. Crompton, J. Phys. B 13, 3451 (1980).

${ }^{10}$ R. J. Gulley, D. T. Alle, M. J. Brennan, M. J. Brunger, and S. J. Buckman, J. Phys. B 27, 2593 (1994).

${ }^{11}$ R. C. Reid, J. M. Prausnitz, and B. E. Poling, The Properties of Gases and Liquids, 4th Ed. (McGraw-Hill, New York, 1987).

${ }^{12}$ K. Rohr, J. Phys. B 10, 2215 (1977).

${ }^{13}$ R. K. Nesbet, Phys. Rev. A 20, 58 (1979). 
${ }^{14}$ See, for example, Y. Itikawa, Phys. Rev. A 3, 831 (1970).

${ }^{15}$ A. A. Radzig and B. M. Smirnov, Reference Data on Atoms, Molecules, and Ions (Springer, Berlin, 1985).

${ }^{16}$ P. D. Burrow, A. Modelli, N. S. Chiu, and K. D. Jordan, J. Chem. Phys. 77, 2699 (1982).

${ }^{17}$ L. Sanche and G. J. Schulz, Phys. Rev. A 5, 1672 (1972).

${ }^{18}$ M. Falcetta and K. D. Jordan, J. Phys. Chem. 94, 5666 (1990).

${ }^{19}$ M. Guerra, D. Jones, D. Distefano, F. Scagnolari, and A. Modelli, J. Chem. Phys. 94, 484 (1991).

${ }^{20}$ G. A. Gallup, J. Phys. B 23, 2383S, 2397S (1990).

${ }^{21}$ G. Herzberg, Molecular Spectra and Molecular Structure (Van Nostrand, Reinhold, New York), Vol. 2; see also, Tables of Molecular Vibrational Frequencies, Natl. Stand. Ref. Data Ser., Natl. Bur. Stand. (U.S.) Circ. No. 39 (U.S. GPO, Washington, DC, 1972).
${ }^{22} \mathrm{~A}$ discussion of the selection rules for vibrational excitation through resonances may be found in G. A. Gallup, J. Chem. Phys. 99, 827 (1993).

${ }^{23}$ A. Mann and F. Linder, J. Phys. B 25, 1621 (1992).

${ }^{24}$ I. I. Fabrikant, J. Phys. B 27, 4325 (1994).

${ }^{25}$ J. M. Blatt and V. F. Weisskopf, Theoretical Nuclear Physics (Wiley, New York, 1952).

${ }^{26}$ W. Domcke, Phys. Rep. 208, 97 (1991).

${ }^{27}$ Y. Itikawa, J. Phys. Soc. Jpn. 30, 835 (1971).

${ }^{28} \mathrm{G}$. A. Gallup (unpublished).

${ }^{29}$ A. M. Krzysztofowicz and C. Szmytkowski, J. Phys. B 28, 1593 (1995).

${ }^{30}$ A. Benitez, J. H. Moore, and J. A. Tossell, J. Chem. Phys. 88, 6691 (1988).

${ }^{31}$ H-X. Wan, J. H. Moore, and J. A. Tossell, J. Chem. Phys. 94, 1868 (1991). 